\title{
In-Band Spectrum Sensing in IEEE 802.22 WRANs for Incumbent Protection
}

\author{
Hyoil Kim, Student Member, IEEE, and Kang G. Shin, Fellow, IEEE
}

\begin{abstract}
In cognitive radios, in-band spectrum sensing is essential for the protection of legacy spectrum users, enabling secondary users to vacate channels immediately upon detection of primary users. For in-band sensing, it is important to meet detectability requirements, such as the maximum allowed detection latency and the probability of misdetection and false alarm. In this paper, we propose key techniques for efficient in-band sensing. We first advocate the use of clustered sensor networks, and propose a periodic in-band sensing algorithm that optimizes sensing period and sensing time to meet the detectability requirements while minimizing sensing overhead. The scheme also determines the better of energy or feature detection incurring less sensing overhead at each SNR level, and derives the threshold $a R S S_{\text {threshold }}$ on the average received signal strength of a primary signal above which energy detection is preferred to feature detection. We consider two key factors affecting $a R S S_{\text {threshold: }}$ noise uncertainty and inter-CRN interference. $a R S S_{\text {threshold }}$ appears to lie between $-114.6 \mathrm{dBm}$ and $-109.9 \mathrm{dBm}$ with noise uncertainty ranging from $0.5 \mathrm{~dB}$ to $2 \mathrm{~dB}$, and between $-112.9 \mathrm{dBm}$ and $-110.5 \mathrm{dBm}$ with 1-6 interfering CRNs. We also investigate how strict the detection requirement must be for efficient reuse of idle channels without incurring unnecessary channel switches due to false detection of primaries.
\end{abstract}

Index Terms-Cognitive radio, IEEE 802.22, energy and feature detection, clustered sensor network, spectrum sensing scheduling.

\section{INTRODUCTION}

Cognitive radio (CR) is a key technology for alleviating inefficient spectrum utilization under the current static spectrum allocation policy [2]. In CR networks (CRNs), unlicensed secondary users (SUs) are allowed to opportunistically utilize (or reuse) spectrum bands ${ }^{1}$ assigned to licensed primary users (PUs) as long as they do not cause any harmful interference to the PUs. The channels reused by the SUs are referred to as in-band channels and all others are called out-of-band channels.

One of the major challenges in CRNs is to protect PUs against interference from SUs for which spectrum sensing is essential. Spectrum sensing monitors: 1) out-of-band channels to determine if they are reusable by detecting PU signals (called out-of-band sensing), and 2) in-band channels to detect return of the PUs so that SUs can promptly vacate the channel in which PUs reappear (called in-band sensing).

For maximal protection of PUs, the FCC has set a strict guideline on in-band sensing. For example, in IEEE 802.22 Wireless Regional Area Networks (WRANs), the world's first international CR standard, PUs should be detected within $2 \mathrm{~s}$ of their appearance with the probabilities of misdetection $\left(P_{M D}\right)$ and false detection $\left(P_{F A}\right)$ no greater than 0.1 . To meet these requirements, in-band sensing must be run frequently enough (at least once every $2 \mathrm{~s}$ ) and a detection method (e.g., energy or feature detection [3]) that

1. We will use the terms spectrum band and channel interchangeably.

- The authors are with the Real-Time Computing Laboratory, Department of Electrical Engineering and Computer Science, The University of Michigan, 2260 Hayward Street, Ann Arbor, MI 48109-2121.

E-mail: \{hyoilkim,kgshin\}@eecs.umich.edu.

Manuscript received 16 Aug. 2009; revised 13 Jan. 2010; accepted 19 Jan. 2010; published online 2 Sept. 2010.

For information on obtaining reprints of this article, please send e-mail to: tmc@computer.org, and reference IEEECS Log Number TMC-2009-08-0342. Digital Object Identifier no. 10.1109/TMC.2010.169. yields the best performance should be selected. The impact of spectrum sensing on SUs' quality-of-service (QoS) impairment should also be considered since sensing is performed during quiet periods [3], [4], within which communications between SUs are suspended.

In this paper, we present several techniques for efficient in-band sensing in IEEE 802.22. We first advocate use of clustered sensor networks and identify its unsolved research issues. We then show how to schedule in-band sensing in order to enhance both sensing performance and SUs' QOS in CRNs. Finally, we investigate how strict detection requirements should be, in order to avoid unnecessary channel switches due to false detection of incumbents.

\subsection{Sensor Clustering}

Collaborative sensing [5] is known to be essential for accurate detection of PUs as it exploits sensor diversity via simultaneous sensing of a channel at multiple locations. Presence/absence of PUs on a channel is determined by data fusion of the simultaneous measurements, and OR-rule [6] is the most common fusion rule under which a channel is considered occupied by PUs if at least one sensor reports so. Its sensing performance with $N$ cooperative sensors has been shown as follows:

$$
P_{M D}(N)=\left(P_{M D}\right)^{N} \quad \text { and } \quad P_{F A}(N)=1-\left(1-P_{F A}\right)^{N},
$$

under the assumption that every sensor has the same $P_{M D}$ and $P_{F A}$ for a given signal.

Equation (1), however, does not hold in a large CRN such as the IEEE 802.22 WRAN in which a base station (BS) covers an area of radius ranging from $33 \mathrm{~km}$ (typical) to $100 \mathrm{~km}$ [3]. In such a case, the average received signal strength $^{2}$ (RSS) of a primary signal at two distant sensor

2. Note that the average received signal strength is given by empirical large-scale path loss in the shadow fading channel model in [7]. 


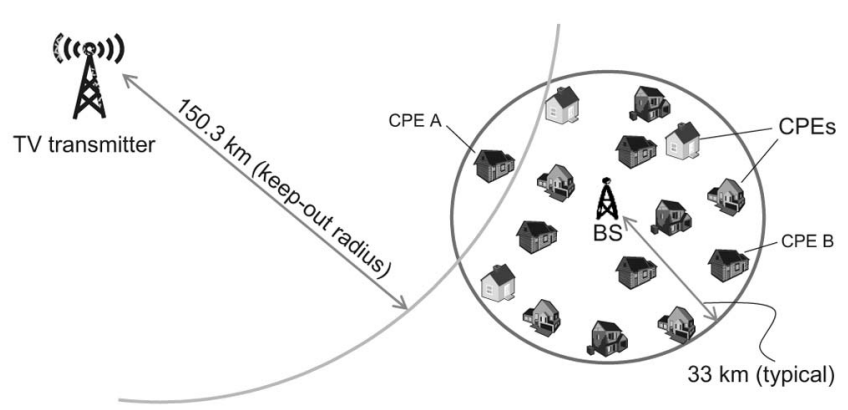

Fig. 1. Illustration of an IEEE 802.22 WRAN.

locations (e.g., CPE A and CPE B in Fig. 1) may vary greatly. Therefore, the heterogeneity of sensors' $P_{M D}$ and $P_{F A}$ values must be considered in analyzing the performance of collaborative sensing, making it harder to determine how many and which sensors are needed to achieve the target sensing performance.

This problem can be avoided or alleviated by using a clustered sensor network, which groups sensors in close proximity into a cluster so that they can acquire a similar average RSS of any primary signal and thus (1) can still hold. Sensor clustering also mitigates the control overhead in data fusion, because each cluster head $(\mathrm{CH})$ makes a local decision based on intracluster (i.e., local) measurements and then reports it to the BS.

Contributions. Although there has been considerable research into clustered CR sensor networks [8], [9], [10], two important issues have not yet been addressed: 1) cluster area size and 2) sensor density. Section 3 addresses these two issues as follows: First, we will derive the maximum radius of a cluster's area so as to upper bound the variation of the average RSS within a cluster by $1 \mathrm{~dB}$. Second, we will derive the maximum sensor density to guarantee nearindependent sensor observations, by suppressing the shadow-fading correlation [11] to be below 0.3. With this approach, one-time (collaborative) in-band sensing in a cluster can be effectively captured by (1) with $N$ as the number of sensors within the cluster.

\subsection{Scheduling of In-Band Sensing}

IEEE 802.22 provides the two-stage sensing (TSS) mechanism that selectively uses energy and feature detection in a quiet period. Although energy detection requires less sensing time (e.g., $1 \mathrm{~ms}$ ), its susceptibility to noise uncertainty [12] limits its usability. By contrast, feature detection is less susceptible to noise uncertainty [13], while it requires a larger sensing time (e.g., $24.2 \mathrm{~ms}$ [3]).

The current IEEE 802.22 draft standard, however, does not specify how often to schedule sensing and which detection method to use, and under what condition. Although there have been several studies on the performance of energy and feature detection [14], [15], [16], they were all based on a onetime detection. Hence, we will study how to enhance detection performance by scheduling sensing and investigate which of energy and feature detection is preferred under what conditions.

Contributions. In Section 4, we propose periodic sensing scheduling that 1) minimizes sensing overhead by optimizing the sensing period ( $T_{P}$ in Fig. 2$)$ and the sensing time ( $T_{I}$

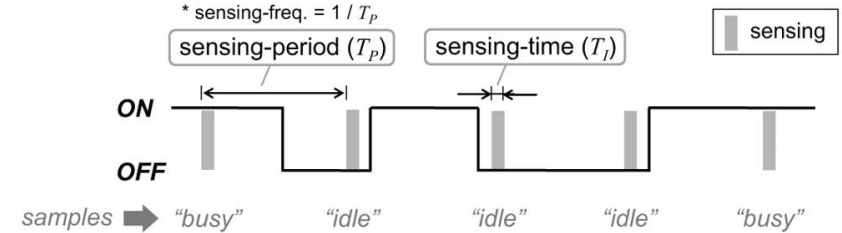

Fig. 2. The ON/OFF channel model and periodic sensing process with sensing period $T_{P}$ and sensing time $T_{I}$.

in Fig. 2), and 2) chooses the better of energy or feature detection in a given sensing environment. Then, in Section 5, we consider $S N R_{\text {wall }}$ [12], [13], the minimum SNR threshold due to noise uncertainty below which a detector completely fails to detect a signal regardless of the sensing time. Although it has been believed that the $S N R_{\text {wall }}$ of energy detection is an absolute barrier, we will show it is true only with the AWGN channel while in reality the barrier becomes obscure with the shadowing channel, making the energy detection still a good candidate due to its small $T_{I}$. Finally, two important factors affecting the detection performancenoise uncertainty and inter-CRN interference-will be considered in deriving two SNR thresholds above which energy detection becomes 1) feasible to use and 2) preferred to feature detection. We also derive the minimum number of collaborative sensors required for feasible energy detection.

\subsection{False Detection versus Efficient Channel-Reuse}

The current $802.22 \mathrm{draft}$ requires the detection performance should satisfy $P_{M D} \leq 0.1$ and $P_{F A} \leq 0.1$, where their purposes on spectrum reuse are vastly different. First, the constraint on $P_{M D}$ focuses on the protection of PUs by encouraging prompt and accurate detection of the returning PUs. On the contrary, the constraint on $P_{F A}$ aims to enhance the QoS of SUs by avoiding impatient channel switching caused by false detection of PUs.

Despite its importance, the impact of $P_{F A}$ has received less attention. As $P_{F A}$ becomes larger, a CRN will unnecessarily vacate an in-band channel and switch to another channel even at PUs' absence. Once a channel is vacated, it is not allowed to be sensed or reused at least for 10 minutes [17], and hence, the pool of idle channels will soon be depleted as the CRN makes such unnecessary channel switches. This implies that $P_{F A}$ should be set small enough not to impair SUs' operation.

Contributions. In Section 6, we investigate if the current upper bound of 0.1 on $P_{F A}$ is efficient in terms of the expected reuse time of an in-band channel. For this, we express the expected channel-reuse time as a function of $P_{F A}$, and vary the upper bound of $P_{F A}$ from 0.001 to 0.1 to evaluate how much we can enhance the channel-reuse time, and at what cost. Based on these results, we will show that $P_{F A}$ needs to be set to be below 0.001 , not 0.1 , for practically meaningful channel-reuse, and will also show that the new constraint induces similar sensing overhead as in the $P_{F A} \leq$ 0.1 case when both achieving $P_{M D} \leq 0.1$.

\subsection{Related Work}

There have been continuing discussions on use of clustered networks in CRs. Chen et al. [8] proposed a mechanism to form a cluster among neighboring nodes and then 
interconnect such clusters. Pawelczak et al. [10] proposed cluster-based sensor networks to reduce the latency in reporting sensor measurements by designating the cluster head as a local decision maker. Sun et al. [9] enhanced performance by clustering sensors where the benefit comes from cluster and sensor diversities. None of these authors, however, mentioned the importance of optimizing cluster size and sensor density.

Despite numerous existing studies on the performance of one-time signal detection in CRs, the optimal scheduling of in-band sensing has not received much attention. Cordeiro et al. [3] evaluated the performance of fast sensing in 802.22 by scheduling it (1 ms) every $40 \mathrm{~ms}$, but they did not optimize the sensing time and sensing period. Datla et al. [18] proposed a backoff-based sensing scheduling algorithm, but their scheme was not designed for detecting returning PUs in an in-band channel. Hoang and Liang [19] introduced an adaptive sensing scheduling method to capture the trade-off between SUs' data-transmission and spectrum sensing. Their scheme, however, did not focus on protection of in-band PUs.

This paper builds upon our preliminary work presented at ACM MobiCom 2008 [1] by 1) considering the impact of small-scale primaries in sensor clustering (Section 3), 2) deriving the minimum number of sensors required for feasible energy detection (Section 5), and 3) dedicating a new section (Section 6) to evaluation of the efficiency of the current 802.22 detectability requirements in terms of the expected reuse time of an idle channel.

\subsection{Organization}

Section 2 briefly reviews IEEE 802.22 for completeness, followed by our channel model and a summary of spectrum sensing. In Section 3, we first introduce the concept of sensor clustering, and then derive the maximum radius of a cluster as well as the maximum sensor density. Section 4 describes the proposed in-band sensing algorithm that can be used in a cluster. Then, in Section 5, we study if simple energy detection is feasible to meet the detection requirements in a very low SNR environment and under what condition it becomes more efficient than feature detection, considering two important factors: noise uncertainty and inter-CRN interference. Finally, Section 6 investigates how strict detection requirements should be for efficient reuse of an idle channel without incurring too frequent channel switches, and the paper concludes in Section 7.

\section{Preliminaries}

\subsection{IEEE $\mathbf{8 0 2 . 2 2}$}

In this paper, we consider important issues of in-band sensing in IEEE 802.22 WRANs. The IEEE 802.22 WRAN is an infrastructure-based wireless network where a base station (BS) coordinates nodes in a single-hop cell which covers an area of radius ranging from $33 \mathrm{~km}$ (typical) to $100 \mathrm{~km}$. End-users of an 802.22 cell are called consumer premise equipments (CPEs) representing households in a rural area (and hence stationary nodes).

802.22 reuses UHF/VHF bands where three types of primary signals are present: analog TV (ATV), digital TV (DTV), and wireless microphone (WM). Our proposed schemes in this paper mostly consider DTV transmitters as the major source of primary transmission, and their extension for WMs is also discussed. ${ }^{3}$ By considering the minimum Desired-to-Undesired (D/U) signal ratio of $23 \mathrm{~dB}$ and the DTV protection contour of $134.2 \mathrm{~km}$, the keep-out radius of CPEs from the DTV transmitter is given as $150.3 \mathrm{~km}$ [14]. CPEs within this keep-out radius are forced to avoid use of the DTV channel. Fig. 1 illustrates this scenario.

\subsection{Channel and Sensing Model}

A channel is modeled as an ON/OFF source, where an ON period represents the time duration during which PUs are using their channel. Hence, SUs are allowed to utilize the OFF periods of the channel. This model has been used successfully in modeling PUs' channel usage pattern in many applications [20], [21]. Note that a TV band usually shows very long ON and OFF periods (e.g., order of hours) by DTV users.

Spectrum sensing is akin to sampling since it measures a channel's state during sensing time (denoted as $T_{I}$ ) and detects the presence of PU signals at that moment. $T_{I}$ may vary with detection methods (e.g., $<1 \mathrm{~ms}$ for energy detection). Fig. 2 illustrates the ON/OFF channel model and an example periodic sensing process with sensing time $T_{I}$ and sensing period $T_{P}$ presented.

In 802.22, sensing must be performed during a quiet period within which no CPEs are allowed to transmit so that any signal detected by sensors should originate from PUs. The quiet periods have to be synchronized among sensors in the same cell as well as between neighboring cells, which is achieved by exchanging coexistence beacon protocol (CBP) frames [3].

\subsection{Signal Detection Methods}

We briefly overview the detection methods used in IEEE 802.22 , along with their theoretical performance in terms of $P_{M D}$ and $P_{F A}$.

\subsubsection{Energy Detection}

Energy detection is the most popular detection method due to its simple design and small sensing time. Shellhammer et al. [14] analyzed the energy detection of a DTV signal using its discrete-time "PHY-layer" samples, where the signal is sampled by its Nyquist rate of $6 \mathrm{MHz} .^{4}$ The detection threshold $\gamma$ to yield $P_{F A}$ is then

$$
\gamma=N_{d} B\left(1+\frac{Q^{-1}\left(P_{F A}\right)}{\sqrt{M_{s}}}\right),
$$

and $P_{M D}$ with $\gamma$ is given as

$$
P_{M D}=Q\left(\frac{\sqrt{M_{s}}}{P+N_{d} B}\left[\left(P+N_{d} B\right)-\gamma\right]\right),
$$

where $M_{s}$ is the number of samples, ${ }^{5} N_{d}$ the noise power spectral density (PSD), $B$ the signal bandwidth $(6 \mathrm{MHz}), P$ the signal power, and $Q(\cdot)$ the $Q$ function.

3. After ATV to DTV transition, ATV is no more of our interest.

4. The DTV signal ranges from $-3 \mathrm{MHz}$ to $+3 \mathrm{MHz}$ in the baseband.

5. By "sample," we mean a "PHY-layer" sample at the ADC, which has nothing to do with the "MAC-layer" sample indicating "idle" or "busy." That is, the ADC samples a channel $M_{s}$ times during $T_{I}$ at each sensing time. 
Note that the effect of multipath fading is insignificant in detecting a DTV signal due to frequency diversity over the $6 \mathrm{MHz}$ band [13], [14]. Instead, the impact of shadow fading must be considered in the variation of RSS at different sensor locations. Ghasemi and Sousa [22] derived the average performance of energy detection by numerically integrating $P_{M D}$ over the fading statistics.

\subsubsection{Feature Detection}

Feature detection captures a specific signature of a DTV signal, such as pilot, field sync, segment sync, or cyclostationarity [3]. Each feature detector is reviewed briefly for completeness.

ATSC uses 8-VSB to modulate a DTV signal, and an offset of 1.25 is added to the signal which creates a pilot signal at a specific frequency location. The authors of [23] introduced pilot energy detection which filters the DTV signal with a $10 \mathrm{kHz}$ narrowband filter at the pilot's frequency location. They showed that the pilot signal's SNR is $17 \mathrm{~dB}$ higher than the DTV signal's SNR, making it a strong feature to detect.

A DTV data segment starts with a data segment sync of pattern $\{+5-5-5+5\}$. A data field consists of 313 data segments, and the first data segment of each data field is called a field sync segment which contains special pseudorandom sequences: PN511 and PN63. Therefore, segment sync and field sync can be used as unique features to detect. Detectors of such features are introduced in [3], [8], [24], where $P_{M D}$ and $P_{F A}$ are not analytically derived but evaluated only by simulation.

Since the DTV signal is digitally modulated, it shows the cyclostationary feature. The cyclostationary detection of ATSC and DVB-T DTV signals has been studied in [8], [16], [25], where its performance is investigated by simulation because it is mathematically intractable to derive $P_{M D}$ and $P_{F A}$ of cyclostationary detectors for complex modulation schemes (e.g., 8-VSB) [26].

In this paper, we use the pilot energy detector (henceforth called simply "pilot detector") as an illustrative example of the feature detectors to evaluate the trade-offs between energy and feature detection. The pilot detector has an advantage over others since its $P_{M D}$ and $P_{F A}$ have been completely analyzed [23], but other types of feature detectors can also be considered by evaluating their $P_{M D}$ and $P_{F A}$ via simulation at various detection thresholds for which the real DTV signal capture [27] and the sensing simulation model [28] can be utilized.

In [23], $P_{M D}$ and $P_{F A}$ of the pilot detector are derived similarly to energy detection, using a $70 \mathrm{kHz}$ bandpass filter in capturing the pilot signal to overcome uncertainty in the pilot locations and inaccuracy in the local oscillator (LO). Hence, we will use the sampling frequency of $70 \mathrm{kHz}$, instead of $10 \mathrm{kHz}$, in our analysis. Unlike energy detection in a $6 \mathrm{MHz}$ bandwidth, Rayleigh fading becomes a significant factor due to the narrow band of $70 \mathrm{kHz}$, and we thus consider both Rayleigh and lognormal shadow fading to derive $P_{M D}$ and $P_{F A}$.

\section{Spectrum Sensor Clustering}

As discussed in Section 1, sensor clustering can make the behavior of collaborative sensing more predictable and can achieve scalability in collecting measurements for data

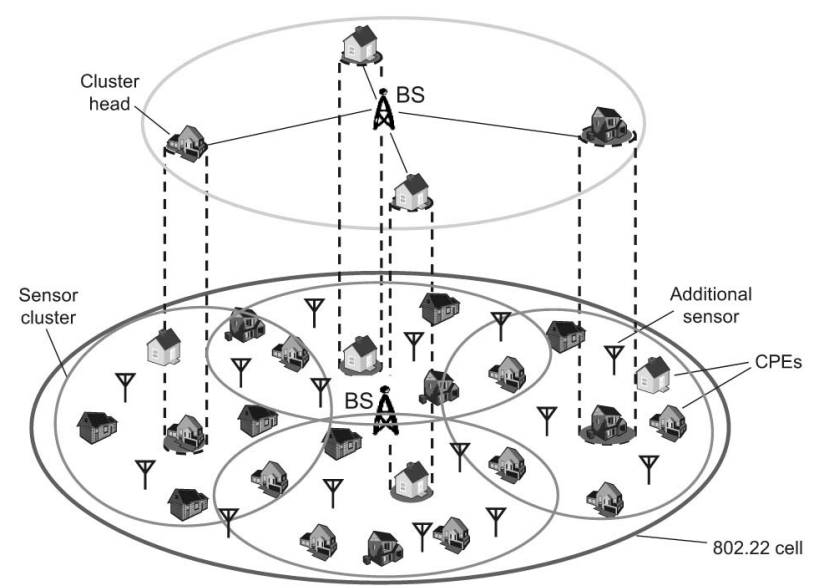

Fig. 3. An illustration of clustered sensor networks.

fusion by enabling cluster heads $(\mathrm{CHs})$ to make local decisions. The concept of the 2-tiered sensor cluster network is illustrated in Fig. 3. In this section, we identify two important but yet-unsolved challenges in sensor clustering: cluster size and sensor density.

\subsection{Cluster Size}

We derive the maximum radius of a sensor cluster such that the variation of the average RSS in the cluster is bounded by $1 \mathrm{~dB}$, to make it possible to use (1) in modeling the performance of a collaborative sensor network. The effect of fading is considered by averaging $P_{M D}$ in (3) over the fading statistics (Rayleigh and/or lognormal) and by substituting it into (1).

In a cluster, the variation of the average RSS is maximized by the two sensors located at $\left(R-R_{c}\right)$ and at $\left(R+R_{c}\right)$ meters away from a primary transmitter (PT), respectively, where $R$ is the distance between the PT and the center of the cluster, and $R_{c}$ is the radius of the cluster. Then, using the polynomial power decay path loss model [29] where the average RSS at a sensor $r$ meters away from the PT is given as $P_{1} r^{-\alpha_{12}}\left(P_{1}\right.$ is the PT's transmit power and $\alpha_{12}$ is the path loss exponent), the maximum cluster size is determined as

$$
10 \alpha_{12} \log _{10}\left(\frac{R+R_{c}}{R-R_{c}}\right) \leq 1(d B)
$$

which gives

$$
R_{c}=\frac{\beta-1}{\beta+1} R, \beta=10^{0.1 / \alpha_{12}} .
$$

For example, for a cluster at the keep-out radius (i.e., $R=150.3 \mathrm{~km}), R_{c}$ is given as $5.76 \mathrm{~km}$, using $\alpha_{12}=3$ suggested in the Hata model [30]. ${ }^{6}$

\subsection{Sensor Density}

We now explore the maximum sensor density to guarantee enough distance between sensors for near-independent

6. Although the Hata model is not the best fit for 802.22 since it is designed for signal propagation up to $20 \mathrm{~km}$ from the transmitter, it works better than the widely accepted Okumura model [31] which does not deal with rural environments. Here, we consider $\alpha_{12}$ as a design parameter and evaluate our schemes with $\alpha_{12}=3$ as an example. Determination of $\alpha_{12}$ is outside of the scope of this paper. 


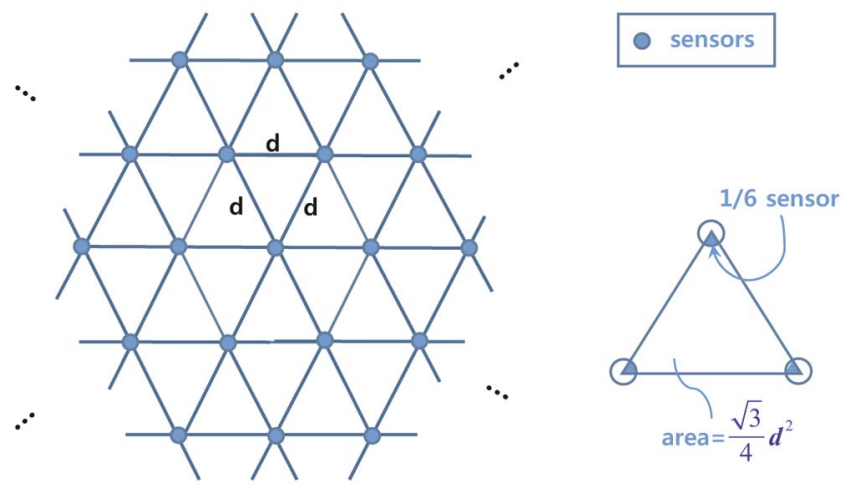

Fig. 4. An example hexagonal sensor deployment.

observations to use (1) for a collaborative sensor network. The need for near-independence also comes from the fact that a few tens of independent sensors provide as much collaborative gain as many more correlated sensors whose collaborative gain is limited by geographical correlation in shadowing [32]. That is, a blind increase in sensor density does not yield a linear increase in collaborative gain.

According to the Gudmundson's model [11], the shadow correlation between two locations that are $d$ meters apart is given as $R(d)=e^{-a d}$ ( $a=0.002$ in a suburban area) which decays exponentially fast. Then, we want to suppress the correlation to be, on average, less than 0.3 between any two neighboring sensors such that $R(d)=e^{-0.002 d} \leq 0.3$ resulting in $d \geq 602 \mathrm{~m}$.

Assuming the hexagonal deployment of sensors as in Fig. 4, where the minimum distance between neighbors is $d$, the density of sensors $\left(D_{S}\right)$ is shown to be

$$
D_{S}=\frac{2}{\sqrt{3} d^{2}}\left(\text { sensors } / \mathrm{m}^{2}\right)
$$

and for $d=602 \mathrm{~m}$, the maximum sensor density is

$$
D_{S}^{\max }=3.18\left(\text { sensors } / \mathrm{km}^{2}\right) .
$$

The minimum sensor density $\left(D_{S}^{m i n}\right)$ is determined by the household density, since a household represents a CPE which plays role as both a sensor and a transceiver. According to the WRAN reference model [33], the minimum household density in a rural area is 0.6 (houses $/ \mathrm{km}^{2}$ ), thus giving

$$
D_{S}^{\min }=0.6\left(\text { sensors } / \mathrm{km}^{2}\right) .
$$

The next question is: at $D_{S}^{\min }$, are there enough (e.g., at least 10) sensors in a cluster for collaboration? Using the above-derived $D_{S}^{\min }$ and $D_{S}^{\max }$, the number of sensors in a cluster ranges between $N_{\text {sensor }}^{\min }$ and $N_{\text {sensor }}^{\max }$ where

$$
N_{\text {sensor }}^{\min }=D_{S}^{\min } \cdot \pi R_{c}^{2}, N_{\text {sensor }}^{\max }=D_{S}^{\max } \cdot \pi R_{c}{ }^{2} .
$$

With $R_{c}=5.76 \mathrm{~km}$, this gives $62-331$ sensors per cluster which exceeds the recommendation in [32]. Therefore, the $\mathrm{CH}$ can select a subset of sensors at each quiet period such that its area can be covered evenly.
TABLE 1

Incumbent Detection Threshold $(I D T)$ of Primary Signals

\begin{tabular}{|c|l|}
\hline Analog TV (NTSC) & $\begin{array}{l}-94 \mathrm{dBm} \text { (at peak of sync of } \\
\text { the NTSC picture carrier) }\end{array}$ \\
\hline Wireless Microphones & $-107 \mathrm{dBm}(200 \mathrm{KHz}$ bandwidth) \\
\hline Digital TV (ATSC) & $-116 \mathrm{dBm}(6 \mathrm{MHz}$ bandwidth) \\
\hline
\end{tabular}

\subsection{Discussion}

\subsubsection{Sensor Locations}

In reality, the location of CPEs may not follow the hexagonal model since they are likely to be cluttered within small areas (e.g., a town or a village) where the actual sensor density is much higher than the average household density (e.g., 0.6 houses $/ \mathrm{km}^{2}$ ). Moreover, CPEs are rare outside the populated areas. Therefore, we take two approaches: 1) the $\mathrm{CHs}$ in a populated area should selectively choose CPEs according to the recommended sensor density to avoid correlated measurements, and 2) additional sensors should be deployed in less-populated areas to achieve $D_{S}^{\min }$ (as shown in Fig. 3). In either case, the hexagonal model may still be useful in selecting proper CPEs for sensing or in deploying more sensors.

\subsubsection{Subclusters}

A sensor cluster may be further divided into smaller subclusters to detect localized deep shadow fading which is not represented well by the lognormal model. Then, more than $N_{\text {sensor }}^{\text {max }}$ sensors can be elected in each cluster to utilize their correlated measurements for identification of the localized shadowing. Further development of subclustering is left as our future work.

\subsubsection{Sensor Clustering for Low-Power Incumbents}

The typical EIRP of wireless microphones (WMs) is $50 \mathrm{~mW}$ in VHF bands and $250 \mathrm{~mW}$ in UHF bands. Due to their use of low power, the footprints of WMs cover a relatively small area compared to high-power primaries, and hence, it is very difficult to find a sufficient number of collaborative sensors in a cluster with identical and independent observations.

To quantify this difficulty, we consider a sensor cluster whose center is $R_{u} \mathrm{~km}$ away from the microphone such that the sensor at its center would experience average RSS of $-107 \mathrm{dBm}$. Here, $-107 \mathrm{dBm}$ is chosen because it is the incumbent detection threshold (IDT) of WMs in IEEE 802.22, where the IDT is the weakest primary signal power (in decibel meter) above which sensors should be able to detect. IDTs for three types of primary signals (in the US) [4] are shown in Table 1 . Then,

$$
R_{u}=\left(10^{-10.7} / P_{u}\right)^{-1 / \alpha_{12}^{\prime}},
$$

where $P_{u}$ is the transmit power of the WM (in milliwatt) and $\alpha_{12}^{\prime}$ is the path loss exponent. For $P_{u}=50 \mathrm{~mW}$

$$
R_{u}= \begin{cases}13.58 \mathrm{~km}, & \text { if } \alpha_{12}^{\prime}=3, \\ 1.25 \mathrm{~km}, & \text { if } \alpha_{12}^{\prime}=4 .\end{cases}
$$


Replacing $R$ and $\alpha_{12}$ in (4) with $R_{u}$ and $\alpha_{12}^{\prime}$ gives

$$
R_{c}= \begin{cases}0.52 \mathrm{~km}, & \text { if } \alpha_{12}^{\prime}=3, \\ 0.036 \mathrm{~km}, & \text { if } \alpha_{12}^{\prime}=4 .\end{cases}
$$

Finally, applying the maximum sensor density $D_{S}^{\max }=3.18$ will result in $2.7\left(\alpha_{12}^{\prime}=3\right)$ and $0.01\left(\alpha_{12}^{\prime}=4\right)$ sensors per cluster. Hence, sensor collaboration is not practical for detecting low-power primaries due to the limited number of available sensors. Fortunately, however, recent work has shown that even a single sensor can meet the detection requirement of wireless microphones by exploiting their special features [34], [35]. So, we will focus on DTV detection via sensor clustering in the remaining part of the paper.

\section{Scheduling of In-Band Sensing}

In this section, we propose an in-band sensing scheduling algorithm that optimizes sensing time and sensing period to achieve detection requirements in IEEE 802.22 with minimal sensing overhead. We first briefly overview the sensing requirements and the two-stage sensing (TSS) mechanism in IEEE 802.22, and then describe the proposed sensing scheduling algorithm with detailed analysis. As mentioned earlier, we will focus on in-band DTV detection.

\subsection{Sensing Requirements in IEEE $\mathbf{8 0 2 . 2 2}$}

Channel detection time $(C D T)$ is given to be $\leq 2 \mathrm{~s}$, within which the returning PUs must be detected with $P_{M D} \leq 0.1$, regardless of the number of times sensing is performed during $C D T$. Similarly, $P_{F A} \leq 0.1$ must also be met when the same sensing algorithm used to meet $P_{M D} \leq 0.1$ is run for $C D T$ seconds during which no PUs are present. The requirement on $P_{M D}$ is to guarantee minimal interference to incumbents, whereas the requirement on $P_{F A}$ is to avoid unnecessary channel switching due to the false detection of PUs.

Based on the above interpretation of $P_{M D}$ and $P_{F A}$, the two performance metrics can be expressed as

$$
\begin{aligned}
P_{M D} & =\operatorname{Pr}\left(\text { detect PT within } C D T \mid H_{1}\right) \leq 0.1, \\
P_{F A} & =\operatorname{Pr}\left(\text { detect PT within } C D T \mid H_{0}\right) \leq 0.1,
\end{aligned}
$$

where $H_{0}$ and $H_{1}$ are two hypotheses on the presence of PUs in the channel:

$$
\begin{aligned}
& H_{0}: \text { No PU exists in the channel, } \\
& H_{1} \text { : PUs exist in the channel. }
\end{aligned}
$$

Note that $P_{M D}$ and $P_{F A}$ in (5) have different meanings from those in (1). $P_{M D}$ and $P_{F A}$ in (5) are the probabilities measured by monitoring an in-band channel for $C D T$ seconds during which sensing may be scheduled multiple times, whereas $P_{M D}$ and $P_{F A}$ in (1) are the probabilities of one-time sensing. To avoid any confusion, we will henceforth replace $P_{M D}$ and $P_{F A}$ in (5) with $P_{M D}^{C D T}$ and $P_{F A}^{C D T}$.

\subsection{TSS Mechanism in IEEE 802.22}

To support a sensing algorithm to meet the detectability requirements shown in (5), IEEE 802.22 provides the twostage sensing (TSS) mechanism. With TSS, a sensing

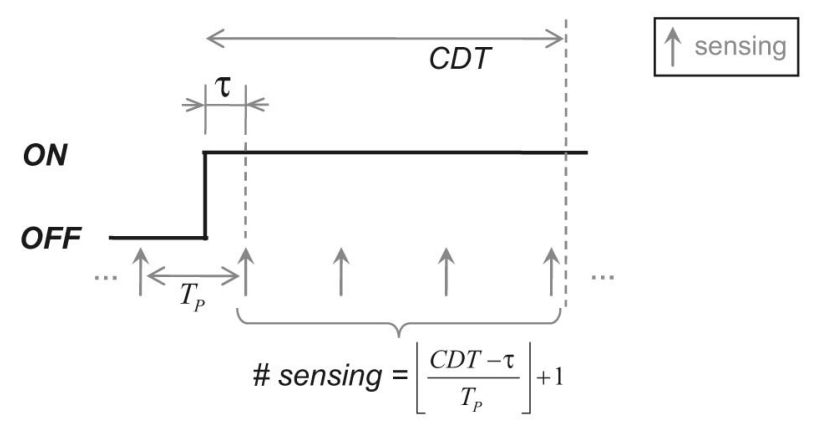

Fig. 5. An example of periodic sensing when a channel transits from OFF to ON due to the returning PUs.

algorithm schedules either fast or fine sensing in each quiet period (QP), where fast sensing employs energy detection while fine sensing uses feature detection.

Although the QPs can be scheduled as many as necessary, there are some restrictions on the sensing period. For example, the QP of fast sensing, usually less than $1 \mathrm{~ms}$, should be scheduled at the end of an 802.22 MAC frame (10 ms) at most once in each frame. Hence, the period of fast sensing becomes a multiple of the frame size (i.e., $n \cdot 10 \mathrm{~ms}$, $n \in N)$. In addition, in case fine sensing adopts a feature detection scheme that requires sensing time longer than one MAC frame (e.g., 24.2 ms for DTV field sync detection), its QP should be scheduled over consecutive MAC frames.

\subsection{In-Band Sensing Scheduling Algorithm}

An efficient sensing algorithm must capture the trade-off between fast and fine sensing, i.e., for one-time sensing: 1) fast sensing consumes a minimum amount of time but its performance is more susceptible to noise uncertainty, and 2) fine sensing usually requires much more time than fast sensing, but its performance is better than fast sensing. Therefore, a sensing algorithm may have to schedule fast sensing at a high frequency, or it may decide to schedule fine sensing at a low frequency. In either case, the scheduling goal is to minimize the overall time spent for sensing (called sensing overhead) while meeting the detectability requirements.

\subsubsection{Analysis of In-Band Sensing Scheduling}

We consider periodic fast or fine sensing. Suppose both fast and fine sensing consume less than $10 \mathrm{~ms}$ (i.e., one MAC frame size) for one-time sensing. Then, the sensing period $T_{P}$ is given as

$$
T_{P}=n \cdot F S, \quad 1 \leq n \leq\left\lfloor\frac{C D T}{F S}\right\rfloor, \quad n \in \mathbb{N},
$$

where $F S$ is the MAC frame size. The upper bound of $T_{P}$ is necessary since sensing must be performed at least once every $C D T$ seconds.

When the channel transits from OFF to ON due to the returning PUs as shown in Fig. 5, periodic sensing will measure the channel $M$ times in CDT seconds, where

$$
M=\left\lfloor\frac{C D T-\tau}{T_{P}}\right\rfloor+1 .
$$


Each (one-time) sensing in Fig. 5 represents collaborative sensing with $N$ sensors whose performance is described by (1). The value of $N$ lies between $N_{\text {sensor }}^{\min }$ and $N_{\text {sensor }}^{\max }$ which were derived in Section 3.

We assume that $\tau / T_{P}$ is uniformly distributed in $[0,1]$ since ON/OFF periods (in the order of hours) are in general much larger than $T_{P}$ (less than $2 \mathrm{~s}$ ). Under this assumption, the probability mass function (pmf) of $M$ is derived as:

$$
\begin{aligned}
& p\left(M_{1}\right)=\operatorname{Pr}\left(M=\left\lfloor\frac{C D T}{T_{P}}\right\rfloor\right)=1-\frac{C D T}{T_{P}}+\left\lfloor\frac{C D T}{T_{P}}\right\rfloor, \\
& p\left(M_{2}\right)=\operatorname{Pr}\left(M=\left\lfloor\frac{C D T}{T_{P}}\right\rfloor+1\right)=\frac{C D T}{T_{P}}-\left\lfloor\frac{C D T}{T_{P}}\right\rfloor .
\end{aligned}
$$

Then, $P_{M D}^{C D T}$ can be expressed as: ${ }^{7}$

$$
\begin{aligned}
P_{M D}^{C D T} & =\sum_{M} \operatorname{Pr}\left(M \text { sensings detect no } \mathrm{PU} \mid H_{1}\right) p(M) \\
& =\sum_{M}\left(P_{M D}(N)\right)^{M} p(M) \\
& =\sum_{M}\left(P_{M D}\right)^{N M} p(M) \leq 0.1 .
\end{aligned}
$$

Similarly, $P_{F A}^{C D T}$ can be expressed as:

$$
\begin{aligned}
P_{F A}^{C D T} & =1-\sum_{M} \operatorname{Pr}\left(M \text { sensings detect no } \mathrm{PU} \mid H_{0}\right) p(M) \\
& =1-\sum_{M}\left(1-P_{F A}(N)\right)^{M} p(M) \\
& =1-\sum_{M}\left(1-P_{F A}\right)^{N M} p(M) \leq 0.1 .
\end{aligned}
$$

In (6) and (7), $P_{M D}$ and $P_{F A}$ are detection-method-specific. They also depend on $T_{I}$ and the RSS of the primary signal. $P_{M D}$ and $P_{F A}$ of energy and pilot detectors are fully described by (2) and (3).

\subsubsection{The Proposed Sensing Scheduling Algorithm}

Our objective is to find the optimal sensing period $T_{P}$ for given $T_{I}$ and RSS that minimizes the sensing-overhead while satisfying two conditions of (6) and (7). The sensing overhead of a sensing algorithm is defined as the fraction of time in which sensing is performed, i.e.,

$$
\text { sensing overhead }=T_{I} / T_{P}
$$

for periodic sensing.

The problem of optimizing $T_{P}$ is identical to that of maximizing $n$ that satisfies (6) and (7). Therefore, the proposed algorithm examines $n$ from its upper bound $\lfloor C D T / F S\rfloor$ and decreases $n$ until the one that meets the condition is found.

Since $P_{F A}^{C D T}$ is a monotonic function ${ }^{8}$ of $P_{F A}$ and there is a one-to-one mapping between $P_{F A}$ and $P_{M D}$, we first want to find the value of $P_{F A}$ that solves the equality of (7). Then, $P_{M D}$ corresponding to $P_{F A}$ can be found from the ROC curve between them. Finally, the feasibility of the tested $n$

7. Here, we assume that $N$ sensors experience the same average RSS, thus having the same $P_{M D}$, since we focus on network planning before deploying sensors without knowledge of actual realization of shadow fading. In case a network designer prefers to use the actual RSS, however, one can extend our equation by introducing $P_{M D}^{s}$, where $s$ is the sensor index.

8. This can be shown by differentiating $P_{F A}^{C D T}$ with respect to $P_{F A}$.

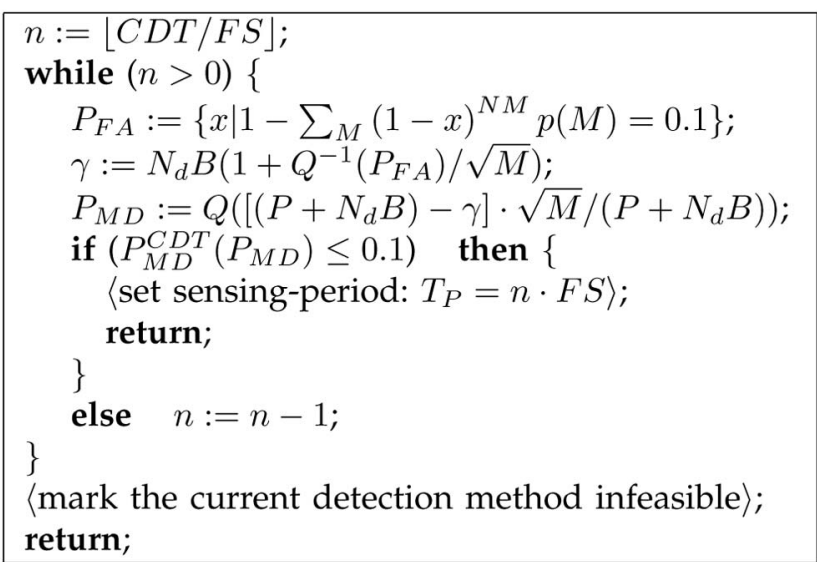

Fig. 6. The in-band sensing scheduling algorithm.

can be checked by substituting $P_{M D}$ into (6). If the tested $n$ does not satisfy (6), then $n$ is decreased by 1 and the above procedure is repeated.

If there does not exist any $n$ satisfying both equations, the detection method considered cannot meet the detectability requirements with given $T_{I}$ and RSS. On the contrary, if the optimal sensing period is found at $n=n_{\text {opt }}$, its sensing overhead is determined as: $T_{I} /\left(n_{\text {opt }} \cdot F S\right)$.

Finally, to find an optimal pair $\left(T_{I}, T_{P}\right)$ that gives the minimal sensing overhead for given RSS, we vary $T_{I}$ within a possible range of interest so that the proposed algorithm finds the best $T_{P}$ for each $T_{I}$. Then, among multiple pairs of $\left(T_{I}, T_{P}\right)$, we choose an optimal pair with minimal sensing overhead. More details on this procedure are provided in Section 5.2.

The pseudocode of the proposed algorithm for energy and pilot detection is given in Fig. 6 .

\subsubsection{Discussion}

An important aspect of the proposed algorithm is that it computes the optimal sensing periods offline, and the optimal periods can be looked up from the database with two inputs, $T_{I}$ and RSS, at runtime. A sensor can create/ store one database per detection method, and adaptively choose the best method with optimal $\left(T_{I}, T_{P}\right)$. In Section 5, we will evaluate and compare the performance of energy and pilot detection. The optimal sensing strategy (i.e., optimal detection method, sensing period, and sensing time) with the average RSS varying from $-120 \mathrm{dBm}$ to $-90 \mathrm{dBm}$ will also be proposed.

In practice, the dissemination of sensing results should also be accounted for. The dissemination delay, however, depends on the type of reporting mechanism used. For example, the dissemination delay can be reduced significantly if the cluster head collects "busy" samples only. Since selecting a reporting mechanism is beyond the scope of this paper, we focus on the detection overhead, but our scheme can be easily extended to include the additional delay by redefining the sensing time as the sum of detection and dissemination times. Moreover, such an additional delay is common to both energy and feature detection, hence keeping unchanged the trade-off between two detection schemes in Section 5. 
We, therefore, focus on the trade-off between energy and feature detection, investigating energy-only and featureonly schemes. As a possible extension, one can consider a hybrid of the two schemes or a more complex dynamic scheduling scheme than simple periodic sensing, which are left as our future work.

\section{Feasibility of Energy Detection}

In this section, we study the feasibility of energy detection in achieving the detectability requirements, and investigate the condition under which energy detection is preferred to feature detection. In addition, we derive the minimum number of sensors necessary for feasible energy detection when the average $R S S$ equals $I D T$.

\subsection{Two Important Factors in In-Band Sensing}

We first briefly overview the impact of noise uncertainty and inter-CRN interference in in-band sensing.

\subsubsection{Noise Uncertainty}

Below $S N R_{\text {wall }}$ [12], energy detection in an AWGN channel completely fails to detect a signal regardless of the sensing time spent. $S N R_{\text {wall }}$ is due to the uncertainty in the noise power (called noise uncertainty) where their relationship is

$$
S N R_{\text {wall }}=\left(\rho^{2}-1\right) / \rho,
$$

when $\rho=10^{x / 10}$ and $x$ is the noise uncertainty in decibel. According to [36], noise uncertainty depends on four factors: calibration error, thermal variation, changes in low-noise amplifier (LNA) gain, and interference. For example, the noise uncertainty under $20^{\circ} \mathrm{K}$ of temperature variation is given as $\pm 1 \mathrm{~dB}$.

Therefore, energy detection is often considered unsuitable for CRNs which must detect a very weak signal power (e.g., as low as $-116 \mathrm{dBm}$ for DTV signals). However, we found that $S N R_{\text {wall }}$ can actually be overcome even when the average SNR (modeled by aRSS) of the collaborative sensors is below $S N R_{\text {wall }}$, since the energy detection of DTV signals will experience a lognormal shadow fading channel, and thus at some sensor locations, instantaneous SNR may exceed $S N R_{\text {wall }}$ due to the location diversity.

Fig. 7a shows the impact of shadow fading, where decibel spread of $5.5 \mathrm{~dB}$ is assumed as in the ITU propagation model of 802.22 [37]. With noise uncertainty of $1 \mathrm{~dB},{ }^{9}$ none of the $N$ sensors overcomes $S N R_{\text {wall }}$ of $-3.33 \mathrm{~dB}$ under the AWGN channel (illustrated as a vertical dotted line at $R S S=-98.5 \mathrm{dBm})$ as predicted in [12]. With shadow fading, however, some sensors under constructive fading ${ }^{10}$ may experience SNR greater than $S N R_{\text {wall }}$ contributing to the performance enhancement, while other sensors under destructive fading do not degrade the performance since their instantaneous RSSs are already below $S N R_{\text {wall }}$ under which $P_{M D}=1$. In addition, Fig. $7 \mathrm{~b}$ shows that the performance of collaborative sensing improves as the number of sensors $N$ increases.

9. We followed the worst-case analysis in [38] where the upper (lower) limit of noise PSD is used to calculate $P_{F A}\left(P_{M D}\right)$, when noise uncertainty is $\Delta \mathrm{dB}$ and the range of noise PSD is given as $-163 \pm \Delta(\mathrm{dBm} / \mathrm{Hz})$.

10. Constructive fading happens under lognormal shadowing, because the instantaneous RSS (in decibel) is modeled as "average RSS (dB) $+X$ $(\mathrm{dB})^{\prime \prime}$ where $X$ is a zero-mean Gaussian random variable.

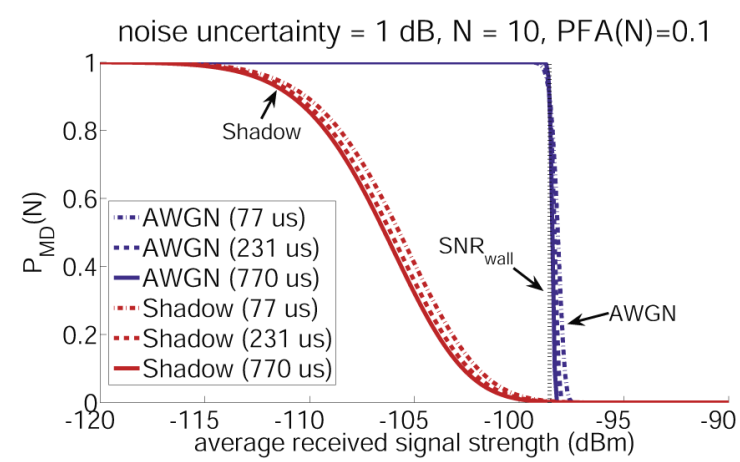

(a)

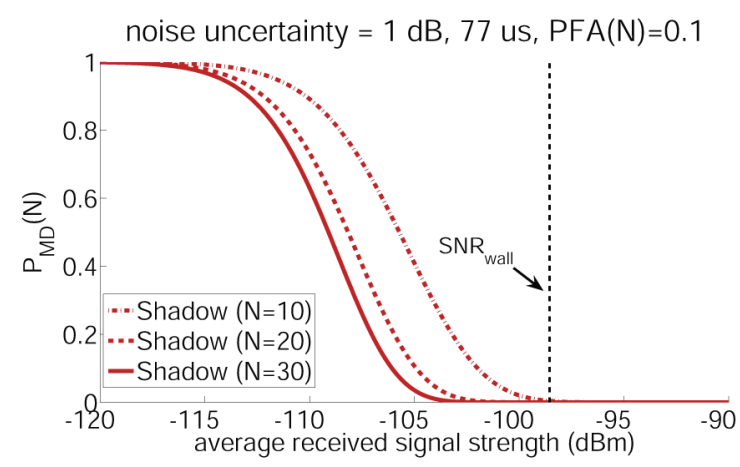

(b)

Fig. 7. Performance comparison (in $P_{M D}(N)$ ) of energy detection: AWGN channel and shadow fading channel. (a) AWGN channel versus shadow-fading channel. (b) Shadow fading channel $\left(T_{I}=77 \mu \mathrm{s}\right)$.

Unlike energy detection, $S N R_{\text {wall }}$ of feature detection decays as the channel coherence time increases [13], meaning that $S N R_{\text {wall }}$ in feature detection is insignificant, since 802.22 CPEs and BSs are stationary devices.

\subsubsection{Inter-CRN Cochannel Interference}

Although the perfect synchronization of QPs between neighboring 802.22 cells is guaranteed by the CBP protocol, 802.22 cells more than one-hop apart may be assigned the same channel. In such a case, they could introduce nonnegligible interference to the CPEs. Moreover, future CRN standards other than IEEE 802.22 may coexist in the same TV bands, which will cause additional interference to 802.22 cells. We call this type of interference inter-CRN cochannel interference.

We first evaluate how much interference is expected between 802.22 cells that are $m$ hops away from each other. Fig. 8 shows two scenarios of cochannel interference. In Fig. 8a, cell A's two-hop neighbor cell B uses the same channel 1, which will interfere with the sensor at the border of cell A. According to [33], a BS with coverage radius of $35 \mathrm{~km}$ will have a transmit EIRP of $23.5 \mathrm{dBW}$, when its antenna has a typical height of $75 \mathrm{~m}$ [39]. The interference power of cell B's $\mathrm{BS}$ to the sensor ${ }^{11}$ is then found to be $-96.5 \mathrm{dBm}$ since

$$
P_{\text {cell B's BS }} \cdot\left(3 R_{\text {cell }}\right)^{-\alpha}=10^{23.5} \cdot\left(3 \cdot 33 \times 10^{3}\right)^{-3} W,
$$

11. Note that the CPEs in cell B are not significant interferers as a CPE uses a directional antenna to communicate with its BS which minimizes its emitted power to the outside of its cell. 
(a)

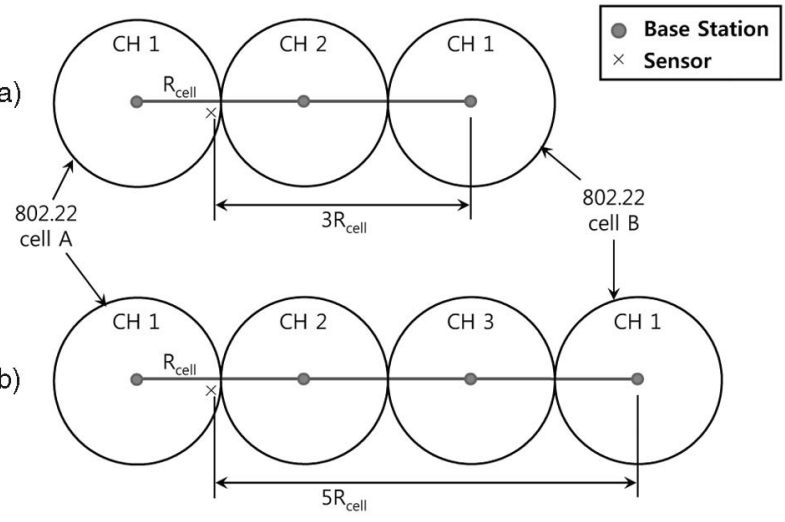

Fig. 8. Intercell interference scenarios in 802.22. (a) 2 hops away. (b) 3 hops away.

which is comparable to the noise power of $-95.2 \mathrm{dBm}$ in the $6 \mathrm{MHz}$ band [14]. In Fig. 8b, however, the three-hop neighbor cell B's interference power is $-103 \mathrm{dBm}$ which is negligible. Hence, we only consider interference from twohop neighbors. Note that additional noise uncertainty due to this inter-CRN interference can be reduced by letting neighboring CRNs exchange the information on their transmission power.

Fig. 9 shows the worst-case scenario of channel assignment for the central 802.22 cell to have maximal inter-CRN interference. There can be up to six two-hop interfering neighbors of a cell. Thus, the interference power will vary from $-\infty \mathrm{dBm}$ (i.e., no interference) to $-88.7 \mathrm{dBm}$ (six times more than $-96.5 \mathrm{dBm}$ ) in our numerical analysis.

\subsection{Optimal Sensing Time and Frequency}

We evaluate energy and pilot detection to find the optimal sensing time $\left(T_{I}\right)$ and sensing period $\left(T_{P}\right)$ to minimize the sensing overhead when they meet the detectability requirements of $P_{M D}^{C D T}, P_{F A}^{C D T} \leq 0.1$.

Each detection scheme is evaluated while varying the average RSS (of the $6 \mathrm{MHz}$ DTV signal) from $-120 \mathrm{dBm}$ to $-90 \mathrm{dBm}$ in steps of $0.1 \mathrm{dBm}$. This RSS range is chosen because 1) the IDT of DTV signal is $-116 \mathrm{dBm}$, and 2) RSS at the keep-out radius of a DTV transmitter is $-96.48 \mathrm{dBm}$ [14].

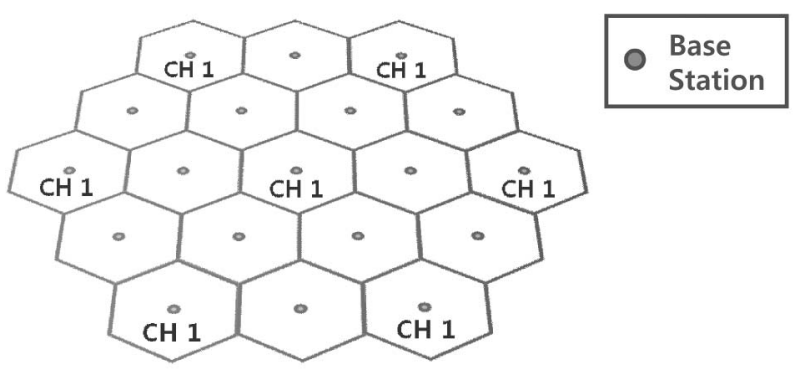

Fig. 9. The worst-case channel assignment to have maximal intercell interference.

Therefore, our interest lies in the range between $-116 \mathrm{dBm}$ and $-96.48 \mathrm{dBm}$, which is well-covered by the simulated RSS range.

We study the impact of noise uncertainty by varying the uncertainty to $0,0.5,1$, or $2 \mathrm{~dB}$. The effect of inter-CRN interference is also evaluated by changing the number of interfering 802.22 cells to $1,2,4$, or 6 cells while fixing noise uncertainty at $1 \mathrm{~dB}$. For both tests, the number of cooperative sensors is fixed at $N=10$.

\subsubsection{Energy Detection}

Since one data segment of a DTV signal is $77 \mu \mathrm{s}$, we tested 10 different sensing times for energy detection, such as $k \cdot(77 \mu \mathrm{s}), k=1,2, \ldots, 10$. During each sensing time, the proposed sensing scheduling algorithm searches for the optimal sensing frequency and the minimal sensing overhead at every RSS value. After optimizing the sensing frequency, the sensing overheads from 10 different sensing times are compared and the best sensing time at each RSS input is chosen.

First, we show the effects of noise uncertainty. Figs. 10a and $10 \mathrm{~b}$ compare energy detection under the noise uncertainty of $0 \mathrm{~dB}$ versus $2 \mathrm{~dB}$. For an illustrative purpose, three sensing times $(1,3$, and 10 segments) are presented. For the $0 \mathrm{~dB}$ case, energy detection performs very well at any RSS with a negligible overhead of less than 0.3 percent. By contrast, for the $2 \mathrm{~dB}$ case, energy detection becomes infeasible for RSS $<-111.7 \mathrm{dBm}$. Note that the blank between $-113 \mathrm{dBm}$ and $-111.7 \mathrm{dBm}$
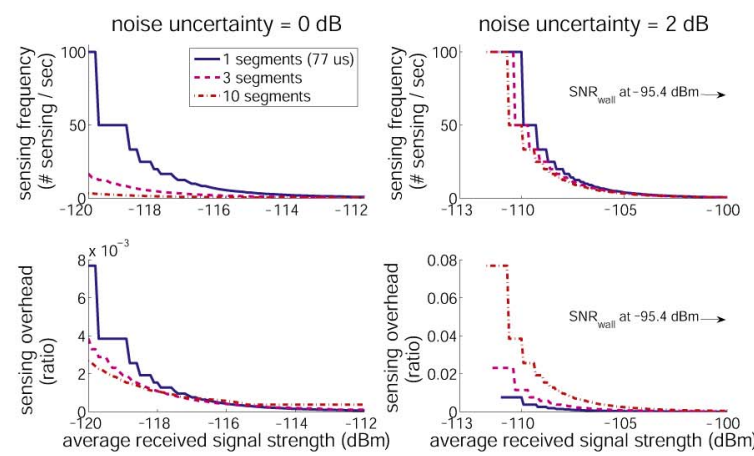

(a)

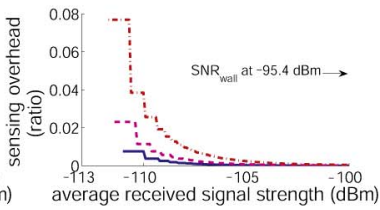

(b)
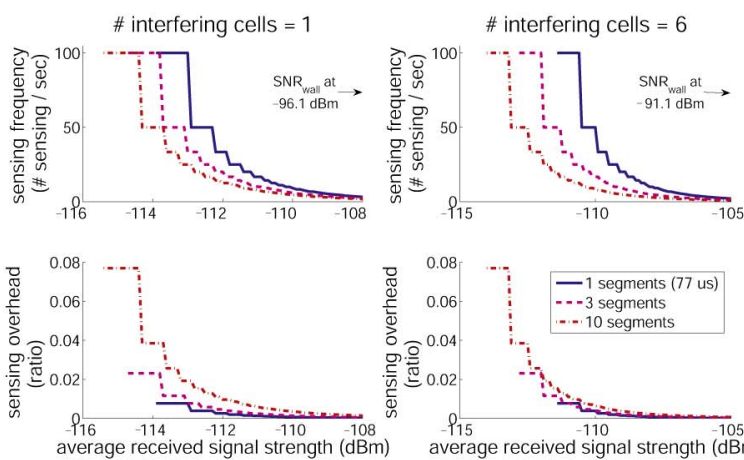

(c)

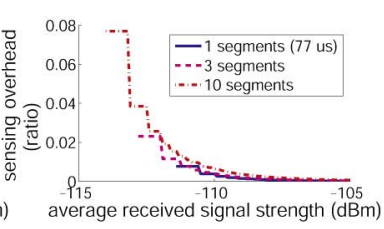

(d)

Fig. 10. Energy detection: sensing overhead and sensing frequency. Various noise uncertainties ((a) $0 \mathrm{~dB}$; (b) $2 \mathrm{~dB})$. Various inter-CRN interferences ((c) 1 cell; (d) 6 cells). 

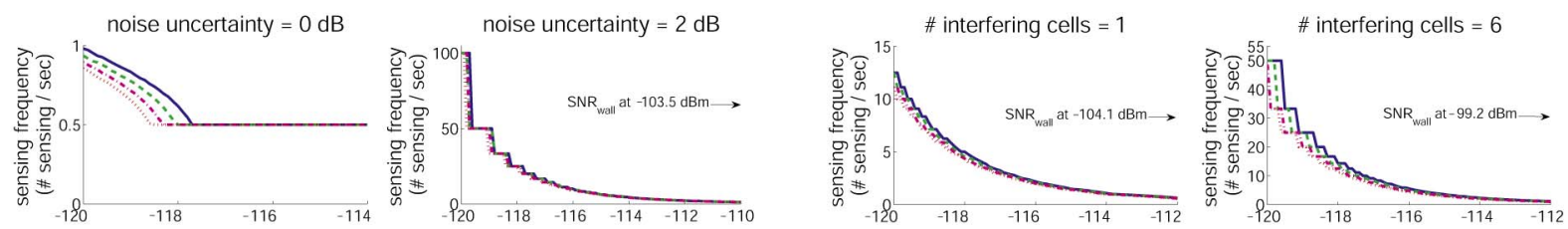

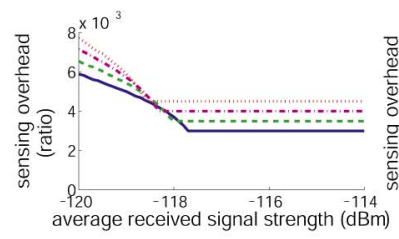

(a)

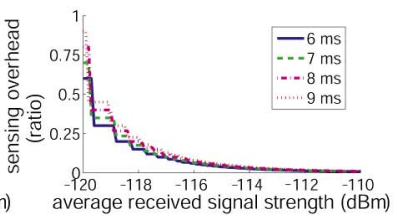

(b)

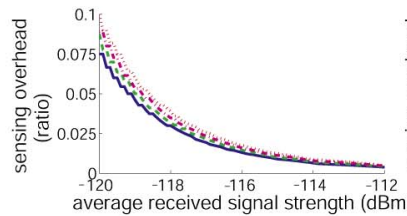

(c)

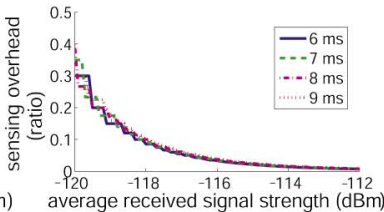

(d)

Fig. 11. Pilot detection: sensing overhead and sensing frequency. Various noise uncertainties ((a) $0 \mathrm{~dB}$; (b) $2 \mathrm{~dB})$. Various inter-CRN interferences ((c) 1 cell; (d) 6 cells).

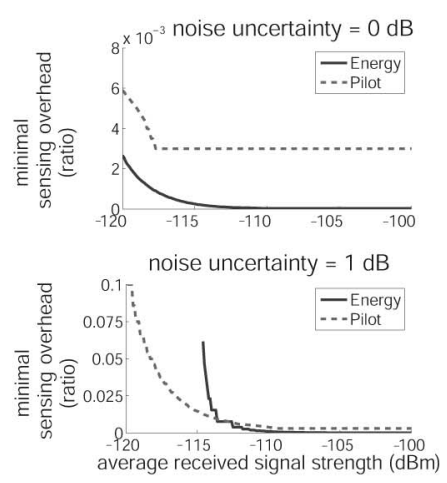

(a)
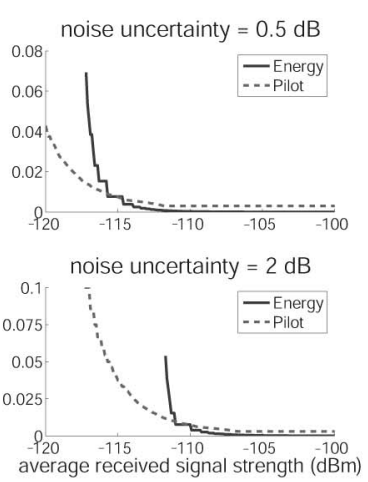
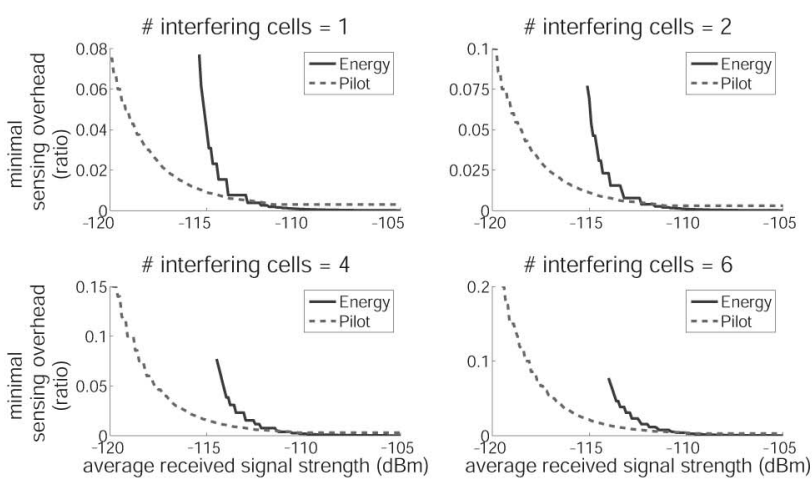

(b)

Fig. 12. Energy detection versus pilot detection: location of $a R S S_{\text {threshold }}$.

(a) Various noise uncertainties. (b) Various inter-CRN interferences.

implies that there is no $T_{P}$ satisfying the detectability requirements. However, compared to the AWGN's $S N R_{\text {wall }}$ of $-95.4 \mathrm{dBm}$, energy detection's feasibility region is enlarged significantly thanks to the sensor diversity under the shadow fading. Interestingly, at the $2 \mathrm{~dB}$ case, the performance (in terms of sensing overhead) does not get better as the sensing time grows, since the impact of $S N R_{\text {wall }}$ becomes more dominant at a larger noise uncertainty.

Second, we vary the number of interfering 802.22 cells to observe the behavior of energy detection. Figs. 10c and 10d show two extreme cases: 1 cell versus 6 cells. As expected, an increase of interfering cells increases the noise plus interference power which impairs performance due to degraded SNR.

\subsubsection{Feature (Pilot) Detection}

Since pilot (energy) detection is based on the energy measurement of a pilot signal, it requires a sufficient number of samples to yield satisfactory results. Due to its lower sampling frequency, sensing time of pilot detection is chosen to be 85 times longer $(6 \mathrm{MHz} / 70 \mathrm{kHz}=85.7)$ than that of energy detection to acquire the same number of samples (i.e., $M_{s}$ ) as energy detection. ${ }^{12}$ On the other hand,

12. Since (2) and (3) are approximated performances by applying the Central Limit Theorem [14], their accuracy degrades as $M_{s}$ decreases. Hence, we match $M_{s}$ by adjusting sensing time, to compare energy and feature detection under the same condition. the MAC frame size of $10 \mathrm{~ms}$ gives an upper bound of sensing time. Based on this observation, we vary the sensing time of pilot detection to be $6,7,8$, or $9 \mathrm{~ms}$, considering that $85.7 \times 77 \mu \mathrm{s}=6.6 \mathrm{~ms}$.

Figs. 11a and $11 \mathrm{~b}$ plot the performance of pilot detection while varying the noise uncertainty. Note that the $\mathrm{x}$-axis represents the average RSS of a $6 \mathrm{MHz}$ DTV signal, not of a pilot signal in the $70 \mathrm{kHz}$ band. The power of pilot signal is $11.3 \mathrm{~dB}$ less than the DTV signal power. Unlike energy detection, pilot detection is feasible at every RSS regardless of the level of noise uncertainty, due to its higher SNR at the pilot location.

Figs. 11c and 11d show the performance of pilot detection while varying the number of interferers. At a given number of interfering cells, the sensing time does not appear to offer a large performance enhancement.

\subsection{Energy Detection versus Feature Detection}

Finally, we investigate the location of $a R S S_{\text {threshold }}$, below which pilot detection is preferred to energy detection. We also introduce $a R S S_{\min }^{\text {energy }}$, the minimum aRSS above which energy detection becomes feasible for detection of DTV signals.

Fig. 12a compares the minimal sensing overheads of energy and pilot detection under various noise uncertainty conditions. With no noise uncertainty, energy detection is the best to use. As the noise uncertainty grows, however, pilot detection becomes preferable at a low aRSS and 
TABLE 2

RSS Thresholds under Various Noise Uncertainties

\begin{tabular}{|c|c|c|c|}
\hline noise uncertainty & $0.5 \mathrm{~dB}$ & $1 \mathrm{~dB}$ & $2 \mathrm{~dB}$ \\
\hline$a R S S_{\text {threshold }}(\mathrm{dBm})$ & -114.6 & -112.5 & -109.9 \\
\hline$a R S S_{\text {min }}^{\text {energy }}(\mathrm{dBm})$ & -117.2 & -114.6 & -111.7 \\
\hline
\end{tabular}

$a R S S_{\text {threshold }}$ increases accordingly. The position of $a R S S_{\text {threshold }}$ is shown in Table 2 along with aRS $S_{\text {min }}^{\text {energy }}$. With 1 or $2 \mathrm{~dB}$ noise uncertainty, pilot detection becomes feasible and preferable even at $-120 \mathrm{dBm}$, but it incurs more than 10 percent of sensing overhead.

$a R S S_{\text {threshold }}$ and $a R S S_{\min }^{\text {energy }}$ of various inter-CRN interferences are also presented in Fig. $12 \mathrm{~b}$ and Table 3. With 1 or $2 \mathrm{~dB}$ noise uncertainty, pilot detection incurs more than 15 percent of sensing overhead at $-120 \mathrm{dBm}$.

From Tables 2 and 3, one can see that the feasibility region of energy detection is reduced just by $1.4 \mathrm{~dB}$ between 1 cell and 6 cells, whereas the gap is $5.5 \mathrm{~dB}$ between noise uncertainty of 0.5 and $2 \mathrm{~dB}$. As a result, noise uncertainty seems to have a more significant influence on energy detection's performance.

\subsubsection{Other Feature Detectors}

From Figs. 12a and 12b, one can observe that energy detection, above $a R S S_{\text {threshold, }}$ incurs at most 0.385 percent of sensing overhead. Here, we compare this overhead with three other types of feature detectors than pilot energy detection: the pilot location detection in [40], the PN511 detection in [3], and the cyclostationary detection in [15]. Since sensing times for such feature detectors are 30, 24.1, and $19.03 \mathrm{~ms}$, respectively, their sensing overheads are given as at least 1.5, 1.2, and 0.95 percent, respectively, even when sensing is scheduled only once every $C D T$ seconds. Therefore, energy detection performs better in its preferred region (i.e., above $a R S S_{\text {threshold }}$ ) than the pilot energy as well as other three types of feature detectors under consideration.

\subsection{Minimum Number of Sensors for Feasible Energy Detection at $a R S S=I D T$}

As shown in Fig. $7 \mathrm{~b}$, the performance of collaborative sensing improves as $N$ grows. Thus, as we increase $N$, $a R S S_{\min }^{\text {energy }}$ also becomes smaller. This means that we can find a minimum $N$, denoted by $N_{\min }$, with which energy detection can be feasible even at $a R S S=I D T$. Here, we investigate such $N_{\min }$ and its relationship with other optimization parameters, such as $T_{I}$ and $T_{P}$.

Fig. 13 illustrates the trade-off between sensing overhead and $N_{\min }$. As we allow higher sensing overhead by introducing longer $T_{I}$ and smaller $T_{P}$, we can achieve the same target detection performance (i.e., $P_{M D}^{C D T}=0.1$ and $P_{F A}^{C D T}=0.1$ ) with

TABLE 3

RSS Thresholds under Various Inter-CRN Interferences

\begin{tabular}{|c|c|c|c|c|}
\hline \# of interferers & 1 & 2 & 4 & 6 \\
\hline$a R S S_{\text {threshold }}(\mathrm{dBm})$ & -112.9 & -112.3 & -111.3 & -110.5 \\
\hline$a R S S_{\min }^{\text {energy }}(\mathrm{dBm})$ & -115.4 & -115.1 & -114.5 & -114 \\
\hline
\end{tabular}

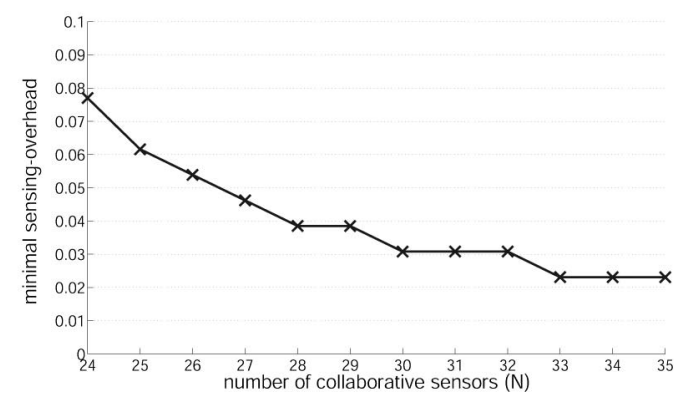

Fig. 13. Number of sensors (i.e., $N$ ) versus optimal sensing overhead $(a R S S=I D T, 1 \mathrm{~dB}$ noise uncertainty).

smaller $N$, and vice versa. Therefore, to find $N_{\min }$, we allow maximum possible overhead, $T_{I}=770 \mu \mathrm{s}$ and $T_{P}=10 \mathrm{~ms}$, in the scenario considered in Section 5.2. Then, the corresponding $N_{\min }$ becomes the lower bound of all possible $N$ s below which no energy detection becomes feasible at $a R S S=I D T$. Table 4 shows the $N_{\min }$ for various noise uncertainties and inter-CRN interferences. In both cases, the maximum $N_{\text {min }}$ (i.e., 221 and 86) does not exceed 331 sensors, which is the maximum number of sensors in a cluster derived in Section 3.

\section{Effective Channel-Reuse Time}

In the current $802.22 \mathrm{draft}$, the detectability requirements are specified as $P_{M D}^{C D T} \leq 0.1$ and $P_{F A}^{C D T} \leq 0.1$. The upper bound of 0.1 on $P_{M D}^{C D T}$ is set for the purpose of protecting PUs, i.e., SUs can only cause limited interference to the PUs returning to the channel currently occupied by the SUs. On the other hand, the upper bound on $P_{F A}^{C D T}$ is set to limit unnecessary channel switches due to false detection of incumbents.

However, the chosen upper bound of $P_{F A}^{C D T}$, denoted by $P_{F A}^{\max }$ (i.e., $P_{F A}^{\max }=0.1$ in the current draft), has not been evaluated for its efficiency in reusing idle channels. Here, we measure the efficiency of $P_{F A}^{\max }$ in terms of the expected duration, $T_{\text {reuse, }}$ of reusing an idle channel until an unnecessary channel switch takes place due to false detection. We focus on this metric because, according to 802.22 , once a channel is vacated due to the detection of PUs in an in-band channel, SUs are not allowed to use the channel again for the Non-Occupancy Period, which is set to 10 minutes [17]. That is, frequent unnecessary channel switches may soon deplete reusable channels even if there are many idle ones.

Thus, we would like to see if the requirement $P_{F A}^{\max }=0.1$ is strict enough by analyzing $T_{\text {reuse }}$ for a given $P_{F A}^{\max }$, and determine a proper $P_{F A}^{\max }$ allowing practically long-enough reuse of idle channels. For this purpose, we consider

TABLE 4

The Minimum Number of Sensors $\left(N_{\min }\right)$ Necessary for Energy Detection to Become Feasible at $a R S S=I D T$

\begin{tabular}{|l|c|c|c|c|}
\hline noise uncertainty & 0 & 0.5 & 1 & 2 \\
\hline$N_{\min }$ & 1 & 5 & 24 & 221 \\
\hline \hline \# of interferers & 1 & 2 & 4 & 6 \\
\hline$N_{\min }$ & 30 & 38 & 58 & 86 \\
\hline
\end{tabular}




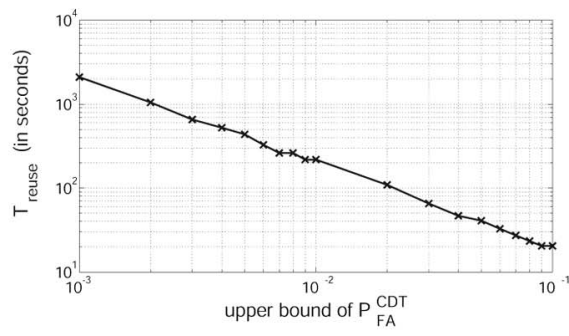

(a)

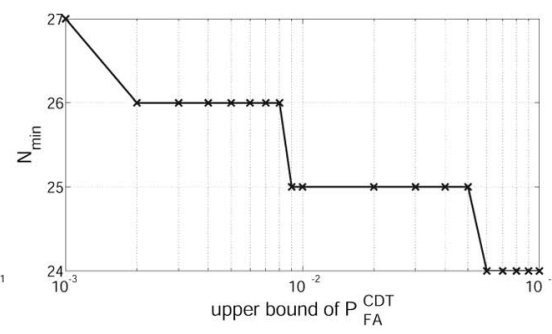

(b)

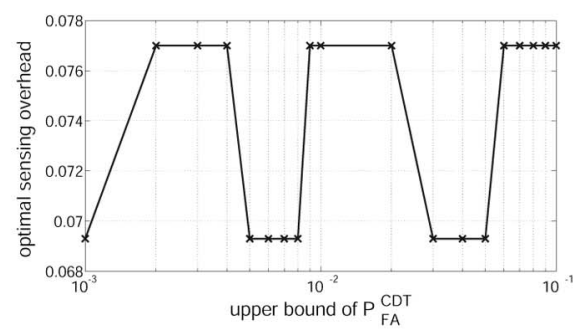

(c)

Fig. 14. Plots of $T_{\text {reuse }}, N_{\text {min }}$, and the optimal sensing overhead $T_{I} / T_{P}$ with varying $P_{F A}^{\max }$. (a) Expected $T_{\text {reuse }}$. (b) $N_{\text {min }}$. (c) Sensing overhead $T_{I} / T_{P}$.

periodic in-band sensing, as in Fig. 5, on an idle channel during its long OFF period, where we assume that each sensing operation is performed by $N$ cooperating sensors with $P_{F A}$ as the single-sensor's false alarm probability.

First, we denote $P_{F A}(N)$ by $P_{F A}^{N}$ for notational simplicity, and express (7) as a function of $P_{F A}^{N}$, i.e., the false alarm probability of single-time cooperative sensing as given in (1), as follows:

$$
P_{F A}^{C D T}\left(P_{F A}^{N}\right)=1-\sum_{M}\left(1-P_{F A}^{N}\right)^{M} p(M)
$$

Then, it is trivial to show that (8) is a monotonic increasing function of $P_{F A}^{N}$. Hence, having $P_{F A}^{C D T}$ upper-bounded by $P_{F A}^{\max }$ is the same as having $P_{F A}^{N}$ upper-bounded by $\hat{P}_{F A}^{\max }$, where $P_{F A}^{C D T}\left(\hat{P}_{F A}^{\max }\right)=P_{F A}^{\max }$.

Next, the expected channel-reuse duration, $T_{\text {reuse, }}$ is derived as

$$
\begin{aligned}
T_{\text {reuse }} & =T_{P} \cdot\left(1-P_{F A}^{N}\right) P_{F A}^{N}+2 T_{P} \cdot\left(1-P_{F A}^{N}\right)^{2} P_{F A}^{N}+\cdots \\
& =\sum_{i=0}^{\infty} i T_{P} \cdot\left(1-P_{F A}^{N}\right)^{i} P_{F A}^{N}=T_{P}\left(\frac{1-P_{F A}^{N}}{P_{F A}^{N}}\right) .
\end{aligned}
$$

Therefore, the smaller $P_{F A}^{N}$, the longer an idle channel can be reused without false detection of PUs. This implies that we need to set $\hat{P}_{F A}^{\max }$ small enough to make $T_{\text {reuse }}$ reasonably large.

We now want to evaluate the efficiency of the current detectability requirement in the 802.22 draft, i.e., $P_{F A}^{\max }=0.1$. While varying $P_{F A}^{\max }$ from 0.001 to 0.1 , we compute $N_{\min }$ for each $P_{F A}^{\max }$ value, where $N_{\min }$ is defined the same as in Section 5.4. Then, for each pair $\left(P_{F A}^{\max }, N_{\min }\right)$, our in-band sensing scheduling algorithm is applied to determine the optimal sensing period $T_{P}$ and sensing time $T_{I}$ with minimal sensing-overhead. Finally, we calculate $T_{\text {reuse }}$ using the chosen $P_{F A}^{\max }$, the derived $T_{P}$, and (9). The results are plotted in Fig. 14.

As shown in Fig. 14a, $T_{\text {reuse }}$ at $P_{F A}^{\max }=0.1$ turns out to be just $20 \mathrm{~s}$. That is, with the currently-chosen upper bound 0.1 , a WRAN can reuse an idle channel for an average of only $20 \mathrm{~s}$ before making an unnecessary channel switch. This implies that a WRAN may require at least 30 idle channels exclusively assigned for its use to provide a seamless service to CPEs, considering the Non-Occupancy Period of 10 minutes. However, there are only 68 channels in TV bands, and thus, it is unlikely to have 30 concurrently idle channels while there are many active TV stations. In addition, self coexistence between neighboring WRANs will make it harder to find an enough number of idle channels reserved for a certain WRAN.

To resolve this issue, we can impose a much more strict upper bound on the false alarm probability by reducing $P_{F A}^{\max }$ significantly. Fig. 14a indicates that by using $P_{F A}^{\max }=0.001$, the expected channel-reuse time is enhanced by two orders-of-magnitude (i.e., $E\left[T_{\text {reuse }}\right]=2,000 \mathrm{~s}$ ) which is reasonably long for SUs to operate on an idle channel. Although one may wonder if the new constraint on $P_{F A}^{\max }$ might increase $N_{\min }$ and the sensing overhead significantly, Figs. $14 \mathrm{~b}$ and $14 \mathrm{c}$ show a minimal increase in $N_{\min }$ (just three more sensors) and a bounded sensing overhead between 0.069 and 0.077 when $P_{F A}^{\max }$ varies.

As a result, we recommend $P_{F A}^{\max }=0.001$ as a new requirement on the false alarm probability. Using this new value of $P_{F A}^{\max }$, combined with the proposed in-band sensing scheduling, we can still meet the detectability requirements with a slight increase in the number of collaborative sensors while enhancing the channel-reuse time significantly.

\section{Conclusion}

In this paper, we discussed three important aspects of inband spectrum sensing in the IEEE 802.22: sensor clustering, sensing scheduling, and detectability requirements. First, we showed the necessity of sensor clustering and derived the maximum sensor cluster size and sensor density. Next, we proposed an in-band sensing scheduling algorithm that minimizes sensing overhead while meeting the detectability requirements, and evaluated its performance with respect to noise uncertainty and inter-CRN interference. We also derived the SNR threshold above which energy detection is preferred to feature detection and the minimum number of collaborative sensors for feasible energy detection at a given SNR. Finally, we investigated how strict the detectability requirement must be to guarantee efficient channel reuse without incurring unnecessarily often channel switches.

In future, we would like to explore subclustering techniques to extend the benefits of using clustered sensor networks. Implementing the proposed algorithms in CR devices should be another step of our plan.

\section{ACKNOWLEDGMENTS}

This research was supported in part by the US National Science Foundation under grants CNS-0519498 and CNS0721529 and in part by the industry grants from Intel 
Corporation, Philips Research North America, and NEC Labs North America. An earlier version of this work was presented at ACM MobiCom 2008 [1].

\section{REFERENCES}

[1] H. Kim and K.G. Shin., "In-Band Spectrum Sensing in Cognitive Radio Networks: Energy Detection or Feature Detection?" Proc. ACM MobiCom, pp. 14-25, Sept. 2008.

[2] FCC, Spectrum Policy Task Force Report, ET Docket No. 02-135, Nov. 2002.

[3] C. Cordeiro, K. Challapali, and M. Ghosh., "Cognitive PHY and MAC Layers for Dynamic Spectrum Access and Sharing of TV Bands," Proc. ACM Int'l Workshop Technology and Policy for Accessing Spectrum (TAPAS '06), Aug. 2006.

[4] IEEE 802.22 Working Group on Wireless Regional Area Networks, http://www.ieee802.org/22, 2010.

[5] G. Ganesan and Y. Li, "Cooperative Spectrum Sensing in Cognitive Radio Networks," Proc. IEEE Symp. New Frontiers in Dynamic Spectrum Access Networks (DySPAN), pp. 137-143, 2005.

[6] A. Ghasemi and E.S. Sousa, "Collaborative Spectrum Sensing for Opportunistic Access in Fading Environments," Proc. IEEE Symp. New Frontiers in Dynamic Spectrum Access Networks (DySPAN), pp. 131-136, Nov. 2005.

[7] A. Goldsmith, Wireless Communications. Cambridge Univ. Press, 2005.

[8] T. Chen, H. Zhang, G.M. Maggio, and I. Chlamtac, "CogMesh: A Cluster-Based Cognitive Radio Network," Proc. IEEE Symp. New Frontiers in Dynamic Spectrum Access Networks (DySPAN), pp. 168178, Apr. 2007.

[9] C. Sun, W. Zhang, and K.B. Letaief, "Cluster-Based Cooperative Spectrum Sensing in Cognitive Radio Systems," Proc. IEEE Int'l Conf. Comm. (ICC '07), pp. 2511-2515, June 2007.

[10] P. Pawelczak, C. Guo, R.V. Prasad, and R. Hekmat, "ClusterBased Spectrum Sensing Architecture for Opportunistic Spectrum Access Networks," IRCTR-S-004-07 Report, Feb. 2007.

[11] M. Gudmundson, "Correlation Model for Shadow Fading in Mobile Radio Systems," Electronic Letters, vol. 27, no. 23, pp. 21452146, Nov. 1991.

[12] R. Tandra and A. Sahai, "Fundamental Limits on Detection in Low SNR Under Noise Uncertainty," Proc. Int'l Conf. Wireless Networks, Comm., and Mobile Computing (WirelessCom), June 2005.

[13] R. Tandra and A. Sahai, "SNR Walls for Feature Detectors," Proc. IEEE Symp. New Frontiers in Dynamic Spectrum Access Networks (DySPAN '07), pp. 559-570, Apr. 2007.

[14] S. Shellhammer, S.N. Shankar, R. Tandra, and J. Tomcik, "Performance of Power Detector Sensors of DTV Signals in IEEE 802.22 WRANs," Proc. ACM Int'l Workshop Technology and Policy for Accessing Spectrum (TAPAS '06), Aug. 2006.

[15] H.-S. Chen, W. Gao, and D.G. Daut, "Spectrum Sensing Using Cyclostationary Properties and Application to IEEE 802.22 WRAN," Proc. IEEE Global Telcomm. (GLOBECOM) Conf., pp. 3133-3138, Nov. 2007.

[16] L.P. Goh, Z. Lei, and F. Chin, "DVB Detector for Cognitive Radio," Proc. IEEE Int'l Conf. Comm. (ICC '07), pp. 6460-6465, June 2007.

[17] C.R. Stevenson, C. Cordeiro, E. Sofer, and G. Chouinard, Functional Requirements for the 802.22 WRAN Standard, IEEE 802.22-05/0007r47, Jan. 2006.

[18] D. Datla, R. Rajbanshi, A.M. Wyglinski, and G.J. Minden, "Parametric Adaptive Spectrum Sensing Framework for Dynamic Spectrum Access Networks," Proc. IEEE Symp. New Frontiers in Dynamic Spectrum Access Networks (DySPAN), pp. 482-485, Apr. 2007.

[19] A.T. Hoang and Y.-C. Liang, "Adaptive Scheduling of Spectrum Sensing Periods in Cognitive Radio Networks," Proc. IEEE Global Telecomm. (GLOBECOM '07) Conf., pp. 3128-3132, Nov. 2007.

[20] H. Kim and K.G. Shin, "Efficient Discovery of Spectrum Opportunities with MAC-Layer Sensing in Cognitive Radio Networks," IEEE Trans. Mobile Computing, vol. 7, no. 5, pp. 533545, May 2008.

[21] S. Geirhofer, L. Tong, and B.M. Sadler, "Dynamic Spectrum Access in the Time Domain: Modeling and Exploiting White Space," IEEE Comm. Magazine, vol. 45, no. 5, pp. 66-72, May 2007.

[22] A. Ghasemi and E.S. Sousa, "Opportunistic Spectrum Access in Fading Channels through Collaborative Sensing," J. Comm., vol. 2, no. 2, pp. 71-82, Mar. 2007.
[23] S. Shellhammer and R. Tandra, An Evaluation of DTV Pilot Power Detection, IEEE 802.22-06/0188r0, July 2006.

[24] S. Shellhammer, An ATSC Detector Using Peak Combining, IEEE 802.22-06/0243r0, Nov. 2006.

[25] N. Han, S. Shon, J.H. Chung, and J.M. Kim, "Spectral Correlation Based Signal Detection Method for Spectrum Sensing in IEEE 802.22 WRAN Systems," Proc. Int'l Conf. Advanced Comm. Technology (ICACT), pp. 1765-1770, Feb. 2006.

[26] A. Sahai and D. Cabric, "Cyclostationary Feature Detection," Tutorial at the IEEE Symp. New Frontiers in Dynamic Spectrum Access Networks (DySPAN '05) (Part II), Nov. 2005.

[27] V. Tawil, DTV Signal Captures, IEEE 802.22-06/0038r0, Mar. 2006.

[28] S. Shellhammer, V. Tawil, G. Chouinard, M. Muterspaugh, and M. Ghosh, Spectrum Sensing Simulation Model, IEEE 802.22-06/ $0028 r 10$, Sept. 2006

[29] A. Sahai, R. Tandra, S.M. Mishra, and N. Hoven, "Fundamental Design Tradeoffs in Cognitive Radio Systems," Proc. ACM Int'l Workshop Technology and Policy for Accessing Spectrum (TAPAS '06), Aug. 2006.

[30] E. Sofer, WRAN Channel Modeling, IEEE 802.22-05/0055r0, July 2005.

[31] T.S. Rappaport, Wireless Communications: Principles and Practices, second ed. Prentice Hall PTR, 2002.

[32] S.M. Mishra, A. Sahai, and R.W. Brodersen, "Cooperative Sensing among Cognitive Radios," Proc. IEEE Int'l Conf. Comm. (ICC), pp. 1658-1663, June 2006.

[33] G. Chouinard, WRAN Reference Model, IEEE 802.22-04/0002r12, Sept. 2005.

[34] H.-S. Chen, W. Gao, and D.G. Daut, "Spectrum Sensing for Wireless Microphone Signals," Proc. IEEE Conf. Sensor, Mesh, and Ad Hoc Comm. and Networks (SECON), June 2008.

[35] S. Xu, Y. Shang, and H. Wang, "SVD Based Sensing of a Wireless Microphone Signal in Cognitive Radio Networks," Proc. Int'l Conf. Comm. Systems (ICCS), pp. 222-226, June 2008.

[36] S. Shellhammer, Numerical Spectrum Sensing Requirements, IEEE 802.22-06/0088r0, June 2006.

[37] S. Shellhammer and G. Chouinard, Spectrum Sensing Requirements Summary, IEEE 802.22-06/0089r1, June 2006

[38] S. Shellhammer and R. Tandra, Performance of the Power Detector with Noise Uncertainty, IEEE 802.22-06/0134r0, July 2006.

[39] W. Caldwell, Draft Recommended Practice, IEEE 802.22-06/0242r04, Mar. 2007.

[40] C. Cordeiro, M. Ghosh, D. Cavalcanti, and K. Challapali, "Spectrum Sensing for Dynamic Spectrum Access of TV Bands," Proc. Cognitive Radio Oriented Wireless Networks and Comm. (CrownCom), July 2007. 


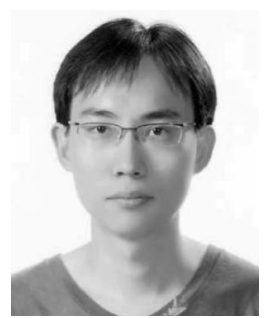

Hyoil Kim received the BS degree in electrical engineering from Seoul National University, Korea, in 1999, and the MS degree from the University of Michigan, Ann Arbor, in 2005 . He is currently working toward the $\mathrm{PhD}$ degree in the Department of Electrical Engineering and Computer Science, University of Michigan. Since 2004, he has been a research assistant at the Real-Time Computing Laboratory in the Electrical Engineering and Computer Science Department, University of Michigan. His research interests include cognitive radios and dynamic spectrum access with an emphasis on network optimization, spectrum sensing, spectrum resource management, quality-of-service, and dynamic spectrum market mechanisms. He was awarded the Korea Science and Engineering Foundation (KOSEF) Scholarship in 2003-2005 and the Samsung Scholarship in 2005-2009. $\mathrm{He}$ is a student member of the IEEE and serves as a peer-reviewer for many IEEE journals and conferences.

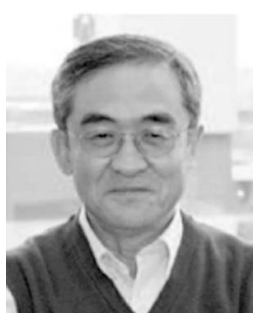

Kang G. Shin received the BS degree in electronics engineering from Seoul National University, Korea, in 1970, and the MS and $\mathrm{PhD}$ degrees in electrical engineering from Cornell University, Ithaca, New York, in 1976 and 1978, respectively. From 1978 to 1982, he was a faculty member of the Rensselaer Polytechnic Institute, Troy, New York. He is the Kevin and Nancy O'Connor professor of computer science and founding director of the Real-Time Computing Laboratory in the Department of Electrical Engineering and Computer Science, University of Michigan, Ann Arbor. His current research focuses on QoS-sensitive networking and computing as well as on embedded real-time OS, middleware, and applications, all with an emphasis on timeliness and dependability. He has supervised the completion of $65 \mathrm{PhD}$ theses and is the author or coauthor of about 700 technical papers (more than 250 of which are in archival journals) and numerous book chapters in the areas of distributed real-time computing and control, computer networking, fault-tolerant computing, and intelligent manufacturing. He has coauthored (jointly with C.M. Krishna) a textbook titled Real-Time Systems (McGraw Hill, 1997). He has received a number of Best Paper Awards, including the IEEE Communications Society William R. Bennett Prize Paper Award in 2003, the Best Paper Award from the IWQoS 2003, and an Outstanding IEEE Transactions on Automatic Control Paper Award in 1987. He has also coauthored papers with his students which received Best Student Paper Awards from the 1996 IEEE Real-Time Technology and Application Symposium and the 2000 UNSENIX Technical Conference. $\mathrm{He}$ has also received several institutional awards, including the Research Excellence Award in 1989, the Outstanding Achievement Award in 1999, the Service Excellence Award in 2000, the Distinguished Faculty Achievement Award in 2001, and the Stephen Attwood Award in 2004 from the University of Michigan; a Distinguished Alumni Award of the College of Engineering, Seoul National University, in 2002; the 2003 IEEE RTC Technical Achievement Award; and the 2006 Ho-Am Prize in Engineering. He has held visiting positions at the US Airforce Flight Dynamics Laboratory, AT\&T Bell Laboratories, the Computer Science Division within the Department of Electrical Engineering and Computer Science at the University of California, Berkeley, the International Computer Science Institute, Berkeley, California, the IBM T.J. Watson Research Center, the Software Engineering Institute at Carnegie Mellon University, and HP Research Laboratories. He also chaired the Computer Science and Engineering Division, Electrical Engineering and Computer Science Department, University of Michigan, for three years beginning January 1991. He is serving as the general cochair for the 2009 ACM Annual International Conference on Mobile Computing and Networking (MobiCom 2009). He was also the general chair for the 2008 IEEE Communications Society Conference on Sensor, Mesh, and Ad Hoc Communications and Networks (SECON 2008), the Third ACM/ USENIX International Conference on Mobile Systems, Applications, and Services (MobiSys 2005), and the 2000 IEEE Real-Time Technology and Applications Symposium (RTAS 2000), the program chair of the 1986 IEEE Real-Time Systems Symposium (RTSS), the general chair of the 1987 RTSS, the guest editor of the 1987 August special issue of the IEEE Transactions on Computers on real-time systems, a program cochair for the 1992 International Conference on Parallel Processing, and has served on numerous technical program committees. He also chaired the IEEE Technical Committee on Real-Time Systems during 1991-1993 and was a distinguished visitor of the IEEE Computer Society, an editor of the IEEE Transactions on Parallel and Distributed Computing, and an area editor of the International Journal of TimeCritical Computing Systems, Computer Networks, and the ACM Transactions on Embedded Systems. He is a fellow of the IEEE and the ACM, and a member of the Korean Academy of Engineering.

$\triangleright$ For more information on this or any other computing topic, please visit our Digital Library at www.computer.org/publications/dlib. 